\title{
Arthroscopic release for painful subtalar stiffness after intra-articular fractures of the
} calcaneum

\author{
K.-B. Lee, \\ J.-Y. Chung, \\ E.-K. Song, \\ J.-K. Seon, \\ L.-B. Bai
}

\section{From Chonnam}

National University

Medical School and Hospital, Gwangiu, Korea

\footnotetext{
K.-B. Lee, MD, PhD, Associate Professor

I. J.-Y. Chung, MD, PhD,

Professor

E.-K. Song, MD, PhD,

Professor

J.-K. Seon, MD, PhD,

Assistant Professor

L.-B. Bai, MD, Researcher

Department of Orthopedic

Surgery

Chonnam National University Medical School and Hospital, 5 HakDong, DongGu, Gwangju 501-757, Korea.
}

Correspondence should be sent to Dr K.-B. Lee; e-mail: kbleeos@chonnam.ac.kr

(C)2008 British Editorial Society of Bone and Joint Surgery doi:10.1302/0301-620X.90B11. $20744 \$ 2.00$

$J$ Bone Joint Surg $[\mathrm{Br}]$ 2008;90-B:1457-61. Received 14 January 2008; Accepted after revision 26 June 2008

We describe the surgical technique and results of arthroscopic subtalar release in 17 patients (17 feet) with painful subtalar stiffness following an intra-articular calcaneal fracture of Sanders' type II or III. The mean duration from injury to arthroscopic release was 11.3 months (6.4 to 36 ) and the mean follow-up after release was 16.8 months (12 to 25). The patient was positioned laterally and three arthroscopic portals were placed anterolaterally, centrally and posterolaterally. The sinus tarsi and lateral gutter were debrided of fibrous tissue and the posterior talocalcaneal facet was released. In all, six patients were very satisfied, eight were satisfied and three were dissatisfied with their results. The mean American Orthopaedic Foot and Ankle Society ankle-hindfoot score improved from a mean of 49.4 points (35 to 66 ) pre-operatively to a mean of 79.6 points (51 to 95 ). All patients reported improvement in movement of the subtalar joint. No complications occurred following operation, but two patients subsequently required subtalar arthrodesis for continuing pain.

In the majority of patients a functional improvement in hindfoot function was obtained following arthroscopic release of the subtalar joint for stiffness and pain secondary to Sanders type II and III fractures of the calcaneum.

Subtalar stiffness is a common complication of intra-articular fracture of the calcaneum. ${ }^{1-3}$ Typically, patients complain of increased pain or discomfort while bearing weight on uneven ground. ${ }^{4}$ Its treatment is difficult. The surgical options include open or arthroscopic subtalar release, but the extensive incision required for the former procedure prohibits early postoperative mobilisation. Lui ${ }^{2}$ described a technique of arthroscopic subtalar release for posttraumatic stiffness which allows early postoperative mobilisation after operation. However, the results have not been reported. We therefore describe the technique of arthroscopic subtalar release in patients with painful subtalar stiffness after treatment for an intra-articular fracture of the calcaneum, and assess the results.

\section{Patients and Methods}

We retrospectively reviewed the results of 17 consecutive arthroscopic subtalar releases undertaken between September 2005 and October 2006. All the patients were male, and their mean age was 42.4 years ( 24 to 56 ). The mechanism of injury involved a fall in 13 patients and a motor vehicle accident in four. Using Sanders' classification $^{5}$ of intra-articular calcaneal fractures 11 patients had a type II fracture and six a type III. The primary treatment had been open reduction and internal fixation with a plate and screws using an extensile lateral approach in type III injuries, and closed reduction and pinning or casting in type II. The mean duration from injury to arthroscopic release was 11.3 months (6.4 to 36), and the mean follow-up after release was for 16.8 months (12 to 25 ) (Table I).

Inclusion criteria were isolated subtalar stiffness with pain on weight-bearing which was aggravated by walking on uneven ground; articular incongruity less than $2 \mathrm{~mm}$ at the posterior facet of the subtalar joint; no narrowing of the joint space or osteophyte formation on plain radiographs and CT scans; and failure to respond to conservative treatment, including pain control with analgesic non-steroidal antiinflammatory medication and functional rehabilitation with physiotherapy.

Radiographs, including CT scans of the ankle, were available for all patients and the American Orthopaedic Foot and Ankle Society (AOFAS) ankle-hindfoot scores $^{6}$ were recorded routinely.

Operative technique. The procedure was performed with patients in the lateral position with the affected foot uppermost under general 
Table I. Results of arthroscopic subtalar release for painful subtalar stiffness after a calcaneal fracture

\begin{tabular}{|c|c|c|c|c|c|c|c|c|c|c|}
\hline \multirow[b]{2}{*}{ Patien } & \multirow[b]{2}{*}{$\begin{array}{c}\text { Age } \\
t \text { (yrs) }\end{array}$} & \multirow[b]{2}{*}{$\begin{array}{l}\text { Sanders' classification } \\
\text { of fracture }\end{array}$} & \multirow[b]{2}{*}{$\begin{array}{l}\text { First treatment }{ }^{*} \text { of } \\
\text { calcaneal fracture }\end{array}$} & \multirow[b]{2}{*}{$\begin{array}{l}\text { Duration }^{\dagger} \\
\text { (mths) }\end{array}$} & \multicolumn{2}{|l|}{ AOFAS $^{\ddagger}$ score } & \multicolumn{2}{|c|}{$\begin{array}{l}\text { Subtalar movement } \\
(\%)\end{array}$} & \multirow{2}{*}{$\begin{array}{l}\text { Cartilage } \\
\text { grade } \\
\text { (ICRS }\end{array}$} & \multirow[b]{2}{*}{$\begin{array}{l}\text { Patient } \\
\text { satisfaction }\end{array}$} \\
\hline & & & & & Pre-operative & Follow-up & \multicolumn{2}{|c|}{ Pre-operative Follow-up } & & \\
\hline 1 & 36 & $\| \mathrm{A}$ & CR and casting & 36.0 & 38 & 87 & 20 & 100 & 1 & Very satisfied \\
\hline 2 & 56 & IIB & $\mathrm{CR}$ and pinning & 18.2 & 52 & 87 & 20 & 80 & 1 & Very satisfied \\
\hline 3 & 34 & IIB & $\mathrm{CR}$ and casting & 14.7 & 38 & 81 & 20 & 80 & 1 & Very satisfied \\
\hline 4 & 53 & IIIAB & ORIF with plate & 14.0 & 44 & 61 & 20 & 60 & 4 & Dissatisfied \\
\hline 5 & 41 & IIB & CR and casting & 22.0 & 59 & 79 & 20 & 80 & 3 & Satisfied \\
\hline 6 & 24 & IIC & CR and pinning & 6.4 & 60 & 81 & 20 & 80 & 2 & Satisfied \\
\hline 7 & 52 & IIB & CR and casting & 32.4 & 46 & 61 & 20 & 40 & 3 & Dissatisfied \\
\hline 8 & 31 & IIIAB & ORIF with plate & 30.3 & 44 & 51 & 10 & 40 & 4 & Dissatisfied \\
\hline 9 & 50 & IIB & $\mathrm{CR}$ and casting & 7.0 & 62 & 85 & 30 & 80 & 2 & Very satisfied \\
\hline 10 & 43 & IIC & $\mathrm{CR}$ and pinning & 17.4 & 41 & 78 & 20 & 80 & 2 & Satisfied \\
\hline 11 & 52 & IIA & $\mathrm{CR}$ and casting & 10.2 & 66 & 95 & 30 & 100 & 1 & Very satisfied \\
\hline 12 & 45 & IIIAB & ORIF with plate & 11.5 & 52 & 84 & 20 & 60 & 2 & Satisfied \\
\hline 13 & 42 & IIIBC & ORIF with plate & 14.5 & 46 & 78 & 20 & 80 & 1 & Satisfied \\
\hline 14 & 45 & IIA & $\mathrm{CR}$ and pinning & 10.9 & 48 & 85 & 30 & 80 & 2 & Satisfied \\
\hline 15 & 41 & IIB & $\mathrm{CR}$ and casting & 7.2 & 44 & 82 & 20 & 80 & 2 & Satisfied \\
\hline 16 & 33 & IIIAC & ORIF with plate & 7.4 & 45 & 83 & 20 & 60 & 2 & Satisfied \\
\hline 17 & 71 & IIIBC & ORIF with plate & 6.5 & 35 & 95 & 30 & 80 & 2 & Very satisfied \\
\hline \multicolumn{11}{|c|}{ * CR, closed reduction; ORIF, open reduction and internal fixation } \\
\hline \multicolumn{11}{|c|}{$\dagger$ duration between initial injury and arthroscopic release } \\
\hline \multicolumn{11}{|c|}{ ‡ AOFAS, American Orthopedic Foot and Ankle Society } \\
\hline \multicolumn{11}{|c|}{$\S$ extent of the range of movement versus normal sides (normal or mild restriction, $76 \%$ to $100 \%$ normal; moderate, $25 \%$ to $74 \%$; marked, $<25 \%$ ) } \\
\hline \multicolumn{11}{|c|}{$\begin{array}{l}\text { II ICRS, International Cartilage Repair Society (grade } 0 \text {, macroscopically normal cartilage; } 1 \text {, cartilage with intact surface but fibrillation, slight soften } \\
\text { ing, superficial laceration and/or fissure; } 2 \text {, defects that extended deeper but involve }<50 \% \text { of the cartilage thickness; } 3 \text {, defects that extended beyond } \\
\text { beyond } 50 \% \text { of the cartilage thickness; } 4 \text {, defects that extended into subchondral bone) }\end{array}$} \\
\hline
\end{tabular}

anaesthesia. After standard preparation and draping, the positions of the lateral malleolus, the intermediate dorsal cutaneous branch of the superficial peroneal nerve, the peroneal tendons and the tendo Achillis were drawn on the skin.

The anterolateral, middle and posterolateral portals for arthroscopy of the subtalar were marked at the estimated sites. $^{7-10}$ A thigh tourniquet was then inflated. The middle portal was established initially, just anterior and distal to the tip of the lateral malleolus, and an 18-gauge needle was used to inject $10 \mathrm{ml}$ of normal saline into the sinus tarsi. A small transverse incision was made through the skin only and a haemostat used to bluntly dissect toward the lateral opening of the sinus tarsi. A $2.5 \mathrm{~mm} 30^{\circ}$ angled arthroscope (Linvatec, ConMed, Largo, Florida) was inserted, and an anterolateral portal was established approximately $1 \mathrm{~cm}$ distal and $2 \mathrm{~cm}$ anterior to the middle portal. Subsequently, a posterolateral portal ${ }^{8-10}$ was created approximately $1 \mathrm{~cm}$ proximal to the tip of the lateral malleolus and just lateral to the tendo Achillis, above the posterosuperior tubercle of the calcaneum (Fig. 1).

During the first stage of the operation, fibrous tissue in the sinus tarsi was debrided using shaver to visualise the anterior and lateral joint line of the posterior subtalar facet. The anterolateral and middle portals were used alternately for viewing and instrumentation.

During the second stage, the lateral gutter (lateral talomalleolar space) was visualised through the anterolateral portal, and lateral subtalar fibrous tissue and impinging soft tissue from the calcaneal fracture were debrided. This was followed by stripping the lateral subtalar capsule and adhesions from the lateral calcaneal cortex with a shaver and small periosteal elevator (Fig. 2). In the majority of cases subtalar movement was substantially recovered at this stage, and the articular surface of the posterior subtalar facet could be examined.

Finally, the posterior portion of the posterior talocalcaneal facet joint was visualised and fibrous tissue was debrided via the posterolateral portal. When this was difficult because of arthrofibrosis, atraumatic distraction was performed using a foot strap. The posterior capsule was then released, and the subtalar joint was passively inverted and everted to improve movement. The extent of subtalar movement was examined under arthroscopic visualisation. The skin incisions were closed with nylon sutures, and a bulky sterile dressing was applied. Active and passive subtalar mobilisation and peroneal strengthening exercises were started on the second post-operative day. The patient was permitted to bear weight as tolerated.

Arthroscopic and clinical evaluation. Post-operative clinical evaluations (by KBL) included assessment of patient satisfaction using a scale of very satisfied, satisfied and dissatisfied, the AOFAS $^{6}$ ankle-hindfoot scores, recovery of subtalar movement and post-operative complications. 


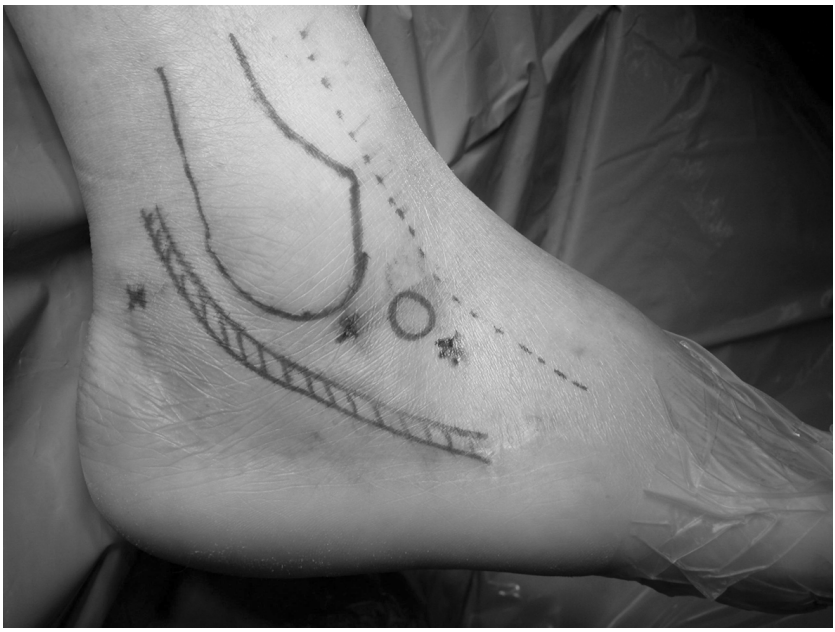

Fig. 1a

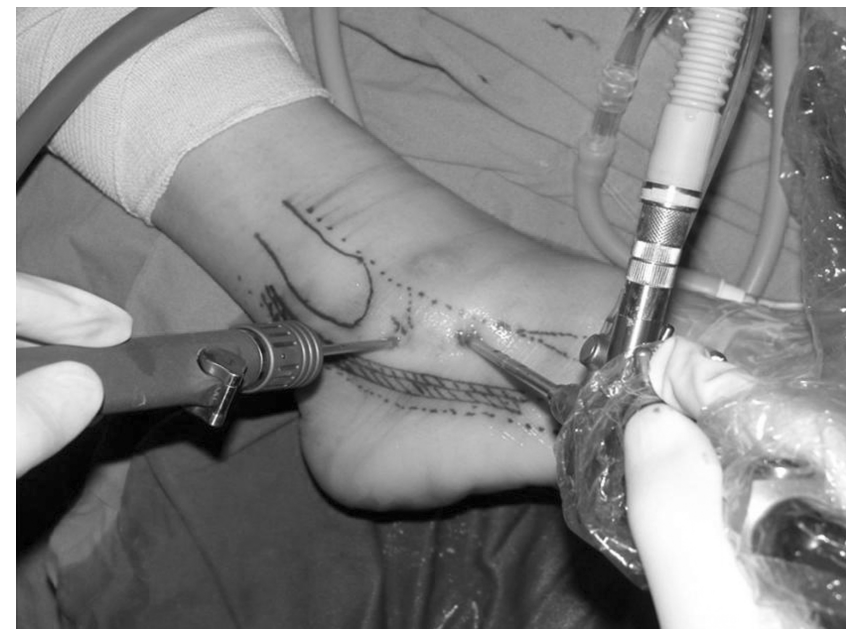

Fig. $1 \mathrm{~b}$

Photograph showing a) anterolateral, middle and posterolateral portals in a patient with an extensive lateral L-shape surgical score from fixation of a calcaneal fracture and b) the arthroscope placed in the anterolateral portal and the shaver in the middle portal to debride fibrous tissue at the sinus tarsi.

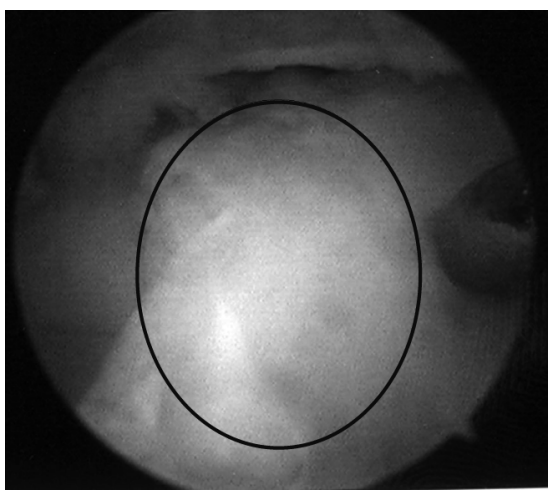

Fig. 2a

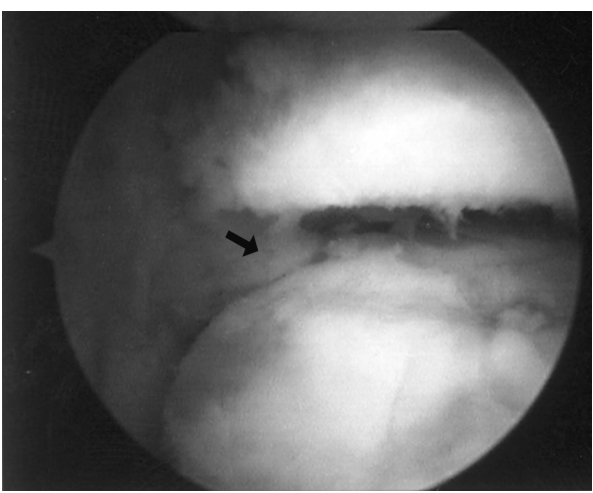

Fig. $2 b$

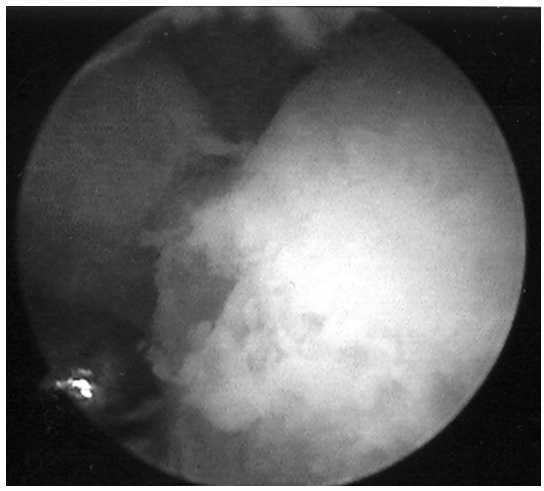

Fig. 2c

Arthroscopic view showing a) fibrous tissue (circle) within the subtalar posterior facet joint, b) impinging lateral subtalar fibrous tissue (arrow), and c) stripping the lateral subtalar capsule and adhesion from the lateral calcaneal cortex with a shaver.

The extent of subtalar movement was determined while the patient was prone on the examining table with their knee flexed to approximately $135^{\circ}$, in which position the axis of the subtalar joint lies close to the horizontal plane. The calcaneum was placed in line with the long axis of the tibia and held in one hand. The heel was then inverted and everted passively while the extent of movement was measured with a goniometer. ${ }^{11}$ The movement of the subtalar joint was described as a percentage of that of the normal side. These values were used to define three groups: a normal joint or mild restriction $(75 \%$ to $100 \%)$, moderate restriction ( $25 \%$ to $74 \%)$, and marked restriction $(<25 \%)$.

The condition of the cartilage of the subtalar joint was rated using the International Cartilage Repair Society (ICRS) classification system, ${ }^{12}$ which focuses on the depth of any lesion and the area of damage.

Statistical analysis. The Mann-Whitney U test was used to evaluate the relationship of the interval between the initial injury ( $<$ one year or $>$ one year) and the arthroscopic release, and the subtalar movement at the last follow-up with clinical outcome. The effect of the grade of injury according to the Sanders' classification ${ }^{5}$ and the method of treatment following injury on the clinical outcome were analysed using the Kruskal-Wallis test. All statistical analyses were performed using SPSS (version 12.0; SPSS Inc., Chicago, Illinois) and were reviewed by an independent statistician.

\section{Results}

The results are shown in Table I. In all, six of 17 patients were 'very satisfied', eight were 'satisfied', and three were 'dissatisfied'. The 14 patients ( $82 \%$ ) who were either very satisfied or satisfied had relief of pain, were able to perform activities of daily living and did not require further local injections or surgical management (Fig. 3). However, pain persisted in the three dissatisfied patients. Two of these 


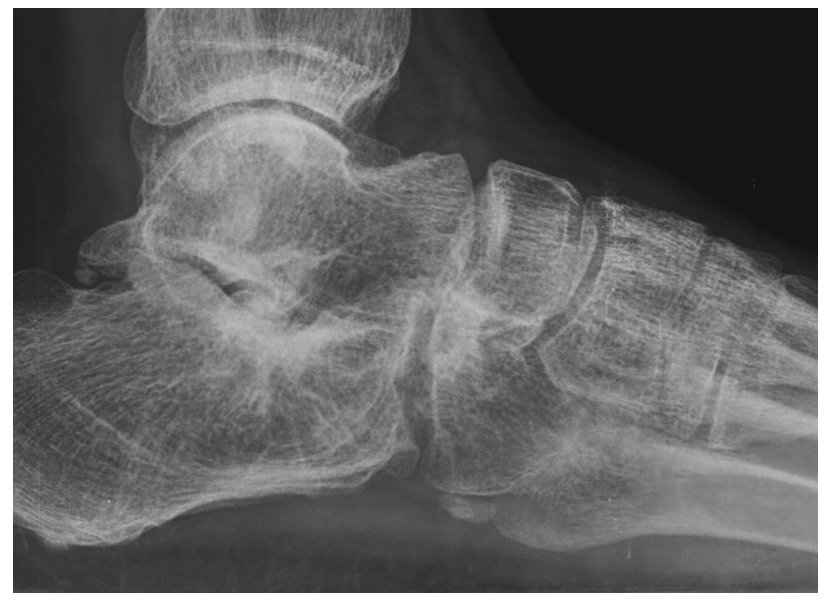

Fig. 3a

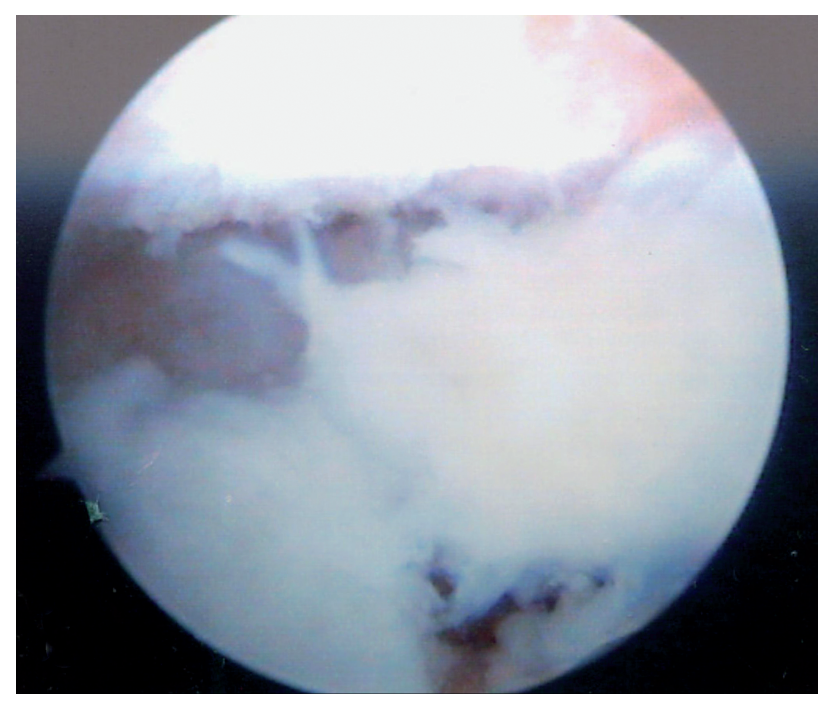

Fig. 3c

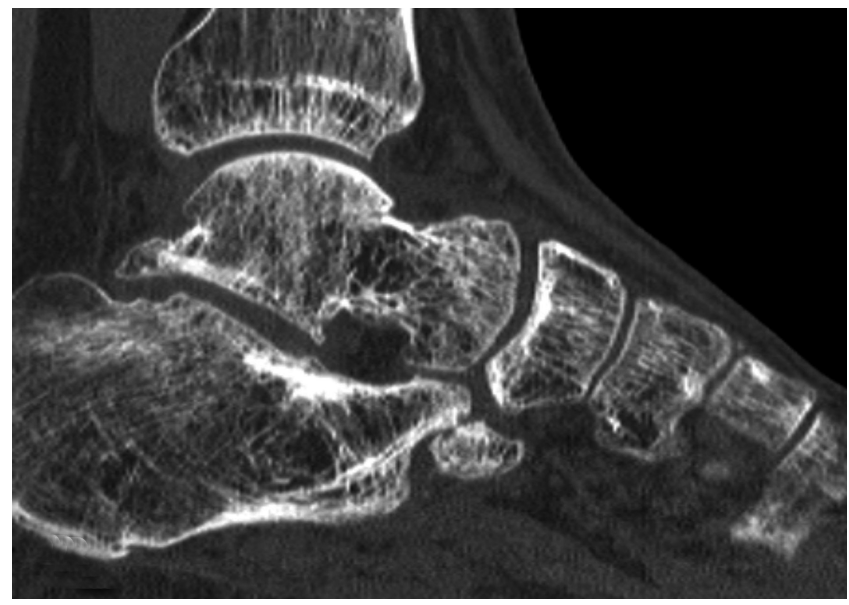

Fig. 3b

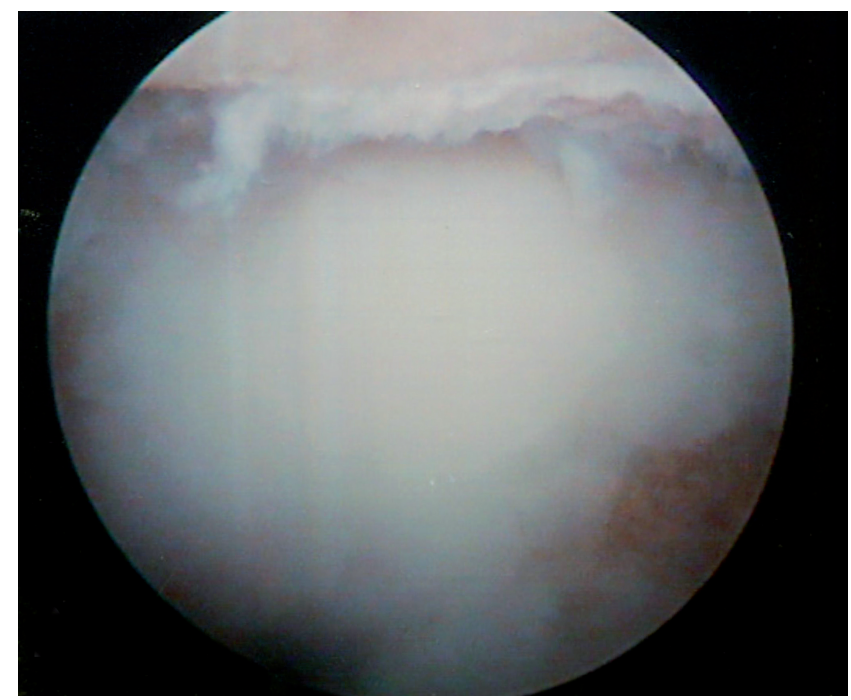

Fig. 3d

a) Lateral radiograph and b) sagittal CT section showing good articular congruity and maintained joint space at the subtalar posterior facet in a 35year-old man with isolated painful subtalar stiffness after calcaneal fracture. Arthroscopic views showing c) fibrous tissue in the sinus tarsi and d) the subtalar joint. Movement improved after release.

(patients 4 and 8) required subsequent subtalar fusion at seven months and 12 months after arthroscopy, which had revealed severe cartilage damage (grade 4).

The mean pre-operative AOFAS $^{6}$ ankle-hindfoot score for all patients was 49.4 points (35 to 66), and this improved to a mean of 79.6 (51 to 95) at final follow-up. At final follow-up the mean AOFAS $^{6}$ score in patients who underwent arthroscopic release within one year of injury was significantly higher than that of those who had the procedure more than one year after injury. The mean AOFAS ${ }^{6}$ score in the eight patients who underwent release within one year of injury improved from a mean of 52 points (35 to 66) to a mean of 86 (81 to 95), and that of the nine patients who underwent release after more than one year after injury improved significantly from a mean of 48 (38 to 59) to a mean of 75 (51 to 87 ) at final follow-up (Mann-Whitney U test; $\mathrm{p}=0.04$ ).

The grades of damage to the subtalar articular cartilage were 1 in six patients, 2 in eight, 3 in one and 4 in two patients. There was a statistically significant relationship between the grades of articular cartilage and the postoperative AOFAS score (Kruskal-Wallis test; $\mathrm{p}=0.049$ ). However, the types of fracture as defined by Sanders' classification $^{5}$ and the initial method of treatment at injury had no significant relationship with clinical outcome (Kruskal-Wallis test; $\mathrm{p}=0.658$ ).

All patients had improvement in movement of the subtalar joint after release. Subtalar joint movement at the last 
follow-up was normal or mildly restricted in ten patients, and moderately restricted in seven. However, no statistical significance was found between patient satisfaction and the degree of recovery of subtalar stiffness (Mann-Whitney test; $\mathrm{p}=0.317$ ).

Post-operatively no patient had a wound infection, neuroma formation or subtalar instability.

\section{Discussion}

Fracture of the calcaneum accounts for $60 \%$ of injuries to tarsal bones and $2 \%$ of all fractures. ${ }^{2,4}$ Intra-articular fractures of the calcaneum have consistently been shown to cause significant disability. ${ }^{5}$ In particular, painful subtalar stiffness may frequently occur. ${ }^{1-3}$ Pozo et al ${ }^{3}$ found that 17 of 21 patients with intra-articular fractures treated nonoperatively had movement of the subtalar joint $<50 \%$ of their normal side. Järvholm et $\mathrm{al}^{1}$ noted that the operative group retained $50 \%$ of normal subtalar joint movement compared to $20 \%$ in the non-operative group. The causes of restricted movement have been attributed to intraarticular arthrofibrosis of the subtalar joint, extra-articular scarring, and adhesion of lateral soft tissues to the lateral calcaneal cortex. ${ }^{2,13}$

In order to restore subtalar movement, vigorous exercises and modifications to footwear during the period of rehabilitation are required. However, when this strategy fails, operative treatment can be considered.

Open subtalar release has the advantage of allowing removal of hardware, but early post-operative mobilisation is prevented by the extensive surgical wound. In contrast, arthroscopic subtalar release is minimally invasive, which facilitates early mobilisation and allows the condition of the posterior calcaneal facet to be examined. ${ }^{2,13-15}$

Parisien and Vangsness ${ }^{8}$ demonstrated that in cases of arthrofibrosis of the subtalar joint dramatic relief of pain and an improved range of movement can be obtained after arthroscopic excision of adhesions. Elgafy and Ebraheim ${ }^{13}$ described ten consecutive patients who underwent subtalar arthroscopy for persistent pain in the subfibular area after open reduction and internal fixation of intra-articular fractures of the calcaneum. Considerable relief of pain was obtained by eight patients, and the two who required subtalar fusion had grade IV degenerative lesions. In 2006, Lui ${ }^{2}$ also described an arthroscopic technique of subtalar release for post-traumatic subtalar stiffness that allows early postoperative mobilisation.

In the present study, 14 patients were either very satisfied or satisfied. In the two patients who required fusion, arthroscopy had revealed grade 4 cartilage damage. Arthroscopic release is a useful procedure for treating painful subtalar stiffness but cannot be relied upon to improve symptoms in patients with severe articular cartilage damage.
All patients had an improvement in movement in the subtalar joint after release. However, satisfaction was not found to be related to the degree of recovery from stiffness. The AOFAS $^{6}$ ankle-hindfoot score in patients who underwent arthroscopic release within one year of injury significantly improved more than that in patients who underwent release more than one year after injury (Mann-Whitney $\mathrm{U}$ test; $p=0.04)$. This suggests that although arthroscopic release plays an important role in the recovery of subtalar movement regardless of the duration between initial injury and release, recovery of movement contributes less to relief of pain in patients who undergo release after a delay exceeding one year from the initial injury.

The limitations of this study are that it lacks randomisation between an open subtalar release and an arthroscopic release, and that no investigation was conducted on the effects of combined lesions, such as fracture of the talus, on the clinical outcomes.

Arthroscopic subtalar release is an invaluable procedure for the treatment of painful subtalar stiffness when conservative treatment fails. The procedure allows early postoperative subtalar mobilisation, improved subtalar joint movement, reduces chronic subtalar pain and improves functional outcome without post-operative morbidity.

No benefits in any form have been received or will be received from a commercial party related directly or indirectly to the subject of this article.

\section{References}

1. Järvholm U, Korner L, Thoren $\mathbf{0}$, Wiklund LM. Fractures of the calcaneus: a comparison of open and closed treatment. Acta Orthop Scand 1984;55:652-6.

2. Lui TH. Arthroscopic subtalar release of post-traumatic subtalar stiffness. Arthroscopy 2006;22:1364.

3. Pozo JL, Kirwan EO, Jackson AM. The long-term results of conservative management of severely displaced fractures of the calcaneus. J Bone Joint Surg [Br] 1984;66-B:386-90.

4. Kingwell S, Buckley R, Willis N. The association between subtalar joint motion and outcome satisfaction in patients with displaced intraarticular calcaneal fractures. Foot Ankle Int 2004;25:666-73.

5. Sanders R. Displaced intra-articular fractures of the calcaneus. J Bone Joint Surg [Am] 2000;82-A:225-50.

6. Kitaoka HB, Alexander IJ, Adelaar RS, et al. Clinical rating systems for the anklehindfoot, midfoot, hallux, and lesser toes. Foot Ankle Int 1994;15:349-53.

7. Frey C, Gasser S, Feder K. Arthroscopy of the subtalar joint. Foot Ankle Int 1994;15:424-8.

8. Parisien JS, Vangsness T. Arthroscopy of the subtalar joint: an experimental approach. Arthroscopy 1985; 1:53-7

9. Amendola A, Lee KB, Saltzman CL, Suh JS. Technique and early experience with posterior arthroscopic subtalar arthrodesis. Foot Ankle Int 2007;28:298-302.

10. van Dijk CN, Scholten PE, Krips R. A 2-portal endoscopic approach for diagnosis and treatment of posterior ankle pathology. Arthroscopy 2000;16:871-6.

11. Coughlin MJ, Mann RA, Saltzman CL. Principles of the physical examination of the foot and ankle. In: Davis WH, Mann RA. Surgery of the foot and ankle. Eighth ed. Philadelphia: Mosby, 2007:45-70.

12. Brittberg M, Winalski CS. Evaluation of cartilage injuries and repair. J Bone Joint Surg [Am]2003;85-A(Suppl 2):58-69.

13. Elgafy H, Ebraheim NA. Subtalar arthroscopy for persistent subfibular pain after calcaneal fractures. Foot Ankle Int 1999;20:422-7.

14. Goldberger MI, Conti SF. Clinical outcome after subtalar arthroscopy. Foot Ankle Int 1998; 19:462-5.

15. Jerosch J. Subtalar arthroscopy: indications and surgical technique. Arthroscopy 1998:6:122-8. 